\title{
The First Eigenvalue of the Laplacian and the Conductance of a Compact Surface
}

\author{
CLARA GRÁCIO ${ }^{1}$ and J. SOUSA RAMOS ${ }^{2, *}$ \\ ${ }^{1}$ Departamento de Matemática, Universidade de Évora, Rua Romão Ramalho, 59, 7000-671 Évora, Portugal; ${ }^{2}$ Departamento \\ de Matemática, Instituto Superior Técnico, Av. Rovisco Pais, 1, 1049-001 Lisboa, Portugal; \\ *Author for correspondence (e-mail: sramos@math.ist.utl.pt; fax: +351-218417035)
}

(Received: 15 September 2004; accepted: 5 April 2005)

Abstract. We present some results with the central theme of is the phenomenon of the first eigenvalue of the Laplacian and conductance of the dynamical system. Our main tool is a method for studying how the hyperbolic metric on a Riemann surface behaves under deformation of the surface. With this model, we show variation of the first eigenvalue of the laplacian and the conductance of the dynamical system, with the Fenchel-Nielsen coordinates, that characterize the surface.

Key words: conductance, Fenchel-Nielsen coordinates, First eigenvalue of the Laplacian, hyperbolic metric, Riemann surface

\section{Introduction}

The Selberg trace formula has been of great interest to mathematicians for almost 50 years. It was discovered by Selberg [1], who also defined the Selberg zeta function, by analogy with the Riemann zeta function, to be a product over prime geodesics in a compact Riemann surface. But the analogue of the Riemann hypothesis is provable for the Selberg zeta function. The trace formula shows that there is a relation between the length spectrum of these prime geodesics and the spectrum of the Laplace operator on the surface.

$$
\begin{aligned}
\operatorname{Tr}\left(e^{t \Delta_{\mathcal{M}}}\right) & =\sum e^{-t \lambda_{k}} \\
& =\operatorname{area}(\mathcal{M}) \frac{e^{-\frac{t}{4}}}{(4 \pi t)^{\frac{3}{2}}} \int_{0}^{+\infty} \frac{b e^{-\frac{b^{2}}{4 t}}}{\sinh \left(\frac{1}{2} b\right)} d b+\frac{1}{2} \sum_{n=1}^{\infty} \sum_{\ell(p)} \frac{\ell(p)}{\sinh \left(\frac{1}{2} \ell\left(p^{n}\right)\right)} \frac{e^{-\frac{t}{4}}}{(4 \pi t)} e^{\frac{-\left(\ell\left(p^{n}\right)\right)^{2}}{4 t}}
\end{aligned}
$$

More recently quantum physicists (specifically those working on quantum chaos theory) have been investigating the Selberg trace formula and its generalizations because it provides a connection between classical and quantum physics. In fact, of late, there has been much communication between mathematicians and physicists on this and matters related to the statistics of spectra and zeta zeros.

The trajectories in deterministically chaotic systems are random. It is therefore no surprise that there are links between the quantum properties of disordered systems and those of simple chaotic systems. The quantum properties of disordered systems have been the focus of considerable attention in many branches of physics. In the last few years, advances in the field of microelectronics have shifted the focus to the study of mesoscopic systems, such as electronic devices that are large on the atomic scale but sufficiently small for quantum coherence effects to be important. In some circumstances the behavior of these devices is governed by the fact that they are disordered. During 


\section{C. Grácio and J. Sousa Ramos}

the last few years work on both the electronic structure of disordered systems and on quantum chaos has been focused on the same fundamental problems, namely localization and properties in energy spectra.

We concentrate our study on techniques based on the trace formula. Over the last few years these questions have led to the development of a theory for the spectral properties of the eigenfunctions of the Laplacian on surfaces of constant negative curvature (on which the geodesic motion is strongly chaotic). The corresponding quantum energy levels are the eigenvalues of the Laplace-Beltrami operator and are related to the closed geodesics by the Selberg trace formula. Physicists also have long studied spectra of Schrödinger operators and the lower value of the energy. Analogously geometers have investigated the first eigenvalue of the Laplacian. Here, using graph theory we study the conductance and the dependence of the spectrum of Laplacian in the deformation parameters of the metric of the space, here a compact surface of constant negative curvature.

For the computation of the length geodesic spectrum, see [2].

In this note, we present a collection of results connected to the behavior of the first eigenvalue $\lambda_{1}(S)$ of the Laplacian on a compact Riemann surface $S$ of genus 2, endowed with a metric of constant curvature -1 .

Our main analytic tool is a method for studying how the hyperbolic metric of a finite-area Riemann surface behaves under such a deformation.

The first eigenvalue of the Laplacian on a surface can be viewed as a functional on the space of Riemannian metrics of a given area.

Every such surface is represented by a quotient space $H^{2} / \Gamma$ of the upper half-plane $H^{2}$ by a Fuchsian group $\Gamma$, which is isomorphic to a fundamental group of $\mathcal{M}$. The discrete group $\Gamma$ is identified with the corresponding system of generators. A fundamental domain $\mathcal{F}$ is defined. The method is to decompose Riemann surface into a set of two pairs of pants by simple closed geodesics. Then the Fenchel-Nielsen coordinates are defined by geodesic length functions of three simple closed geodesics and twist angles along these geodesics.

Here, we use a real-analytic embedding of the Teichmüller space $\mathcal{T}$ of closed Riemann surfaces of genus 2 onto an explicitly defined region $R \subset R^{6}$. The parameters are explicitly defined in terms of the underlying hyperbolic geometry. The parameters are elementary functions of lengths of simple closed geodesics, angles and distances between simple closed geodesics. The embedding is accomplished by writing down four matrices in $\operatorname{PSL}(2, \mathbb{R})$, where the entries in these matrices are explicit algebraic functions of the parameters. With explicit constructions and side pairing transformations (see [2]) we define the Fuchsian group $\Gamma$ representing the closed Riemann surface of genus 2 (Figure 1).

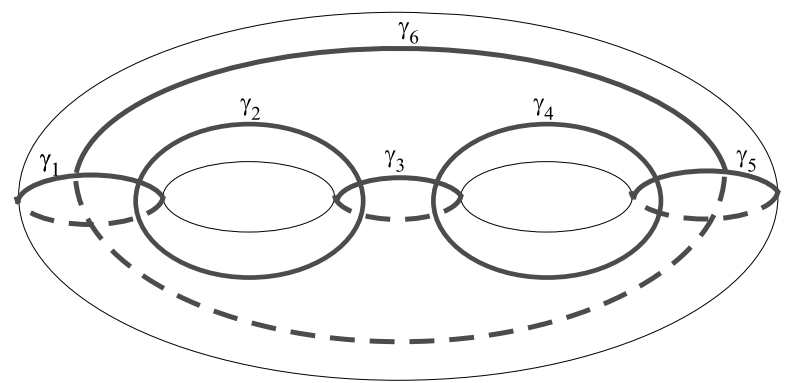

Figure 1. Bitorus: Closed Riemann surface of genus 2. 


\section{Isoperimetric Inequalities}

Cheeger's celebrated inequality relates the first nontrivial eigenvalue of the Laplacian on a compact manifold to an isoperimetric constant, the Cheeger constant, defined as follows: let $S$ be a closed Riemannian manifold (compact, no boundary) and let $X$ be a hypersurface dividing $S$ into two parts, $A$ and $B$. Then

$$
h(S)=\inf _{X} \frac{\operatorname{area}(X)}{\min (\operatorname{vol}(A), \operatorname{vol}(B))}
$$

where the infimum runs over all such hypersurfaces $X$. Let $\lambda_{1}$ denote the first nontrivial eigenvalue of the Laplacian on $S$. Cheeger in [3] proved:

Theorem 1. Let $S$ be a closed Riemannian manifold. Then:

$$
\lambda_{1} \geq \frac{1}{4} h^{2}
$$

This bound is remarkable for its universal character. It is natural to ask whether there is an upper bound for the first eigenvalue in terms of Cheeger's constant. With an added hypothesis on curvatures, such an upper bound was proved by [4]:

Theorem 2. Suppose $S$ is a smooth Riemannian manifold with Ricc $(S) \geq-c$. Then, there are constants $c_{1}$ and $c_{2}$ depending on $c$ so that

$$
\lambda_{1} \leq c_{1} h+c_{2} h^{2}
$$

It is not surprising that there are graph-theoretic analogues of these notions and results.

The idea of studying the first eigenvalue of a Riemann surface via the study of eigenvalues of 3 -regular graphs comes from the work of Buser [4, 5]. In effect, our approach here is a variation on his idea, where we first study the behavior of $\lambda_{1}$ on finite-area Riemann surfaces connected to 3-regular graphs, and then see how $\lambda_{1}$ changes when we deformed the surface. It is interesting to pass back and forth between the geometric and graph-theoretic pictures. One reason for doing this is that a problem which appears difficult from one point of view may be relatively easy, or even solved, from the other point of view. Another reason is that attitudes towards various results may differ markedly in two areas, and comparing them may be an important source of insight.

As usual, there are different conventions and normalizations which are essentially equivalent. It is convenient to stick to the case of $k$-regular graphs $G$, and define the Laplacian as

$$
\Delta(f)(x)=\frac{1}{k} \sum_{y \sim x}(f(x)-f(y))
$$

and the Cheeger constant to be

$$
h(G)=\inf _{X} \frac{\#(X)}{\min (\#(A), \#(B))}
$$


where $X$ runs over collections of edges such that $G-X$ decomposes into two pieces $A$ and $B$, and \# $(A)$ (resp. \#(B)) denotes the number of vertices in $A$ (resp. $B$ ).

Cheeger's inequality then becomes (see [6]):

Theorem 3. Let $\lambda_{1}$ denote the first nontrivial eigenvalue and $h$ the Cheeger constant. Then:

$$
\lambda_{1} \geq \frac{1}{2 k^{2}} h^{2}
$$

The analogue of Buser's inequality is:

Theorem 4. Let $\lambda_{1}$ denote the first nontrivial eigenvalue and $h$ the Cheeger constant. Then:

$$
\lambda_{1} \leq 2 h \text {. }
$$

The importance of the study of the first eigenvalue of a Riemann surface is now fairly well established in the literature. But with the introduction of the notion of conductance of a dynamical system, particularly in the graph theory, and with the relationship with the smallest non-zero eigenvalue of the Laplacian, this importance gets bigger. Another field is hence beginning.

\section{The Notion of Conductance of a Discrete Dynamical System}

In a general way, to each discrete dynamical system $(I, f)$ defined by the iterates of a map $f$ on the interval $I$, we associate a Markov matrix, which is representable by a non-regular, oriented graph $G_{f}$ (the elements of $E$ are now ordered pairs). So we have systems defined by the adjacency matrix $A_{f}=\left(a_{i j}\right)$ of $G_{f}$, that is, the $0-1$ matrix where $a_{i j}=1$ if $i j$ is an edge. We can now establish the notion of conductance of a discrete dynamical system.

In turn, to each $A_{f}$, we associate a probability matrix $P_{f}$ and an invariant measure (the measure of maximal entropy): So we have what is called a random walk in a weighted directed graph, with loops allowed, described by a transition matrix $P_{f}$; which can represent an ergodic system. We can now establish the notion of conductance of a discrete dynamical system.

Definition 1. Let $f$ be a map on the interval, $P_{f}=\left(P_{i j}\right)_{i, j=1}^{n}$ be the probability matrix associated to $f$ and $\pi_{f}=\left(\pi_{i}\right)_{i=1}^{n}$, be the invariant measure. Define conductance of $(I, f)$ by

$$
\Phi_{f}=\min _{\substack{0<\pi(S) \leq \frac{1}{2} \\ S \subset V}}\left(\frac{\sum_{i \in S, j \in \bar{S}} \pi_{i} P_{i j}}{\sum_{i \in S} \pi_{i}}\right)
$$

Another possible approach to the conductance is through the discrete laplacian of a graph defined next.

Definition 2. Let $A_{f}=\left(a_{i j}\right)_{i, j=1}^{n}$ be the adjacency matrix associated to $(I, f)$ and $G_{f}$ the Markov graph. Define the diagonal matrix $D_{f}=\left(d_{i j}\right)_{i, j=1}^{n}$, putting in the diagonal $d_{i i}$ the number of edges that inside (in and out) in the vertex $i$ (loops contribute with 2). We will call the matrix

$$
\Delta_{f}=D_{f}-\left(A_{f}+A_{f}^{T}\right)
$$

the Laplacian matrix of the graph $G_{f}$. 
As we will see, the smallest non-zero eigenvalue of the Laplacian is closely related with the conductance of the system. This result can be proved by symbolic dynamic methods. For more details, see [7].

The notion of the conductance correspond to the notion of Cheeger constant on a Riemann manifold.

\section{Variations with Fenchel-Nielsen Coordinates}

For explicit construction of a fundamental domain we consider the geodesics in the hyperbolic plane $H^{2}$ given the surface $M=H^{2} / \Gamma$, (see Figure 2). When we cut the surface $\mathcal{M}$ along these geodesics then we divide it into four equilateral hexagons. The sides are obtained by the intersection of the axis, they are geodesics segments. These geodesics are the shortest geodesics in the free homotopy class of loops corresponding to some elements $h_{i}(i=1, \ldots, 6)$ of $\Gamma=\pi_{1}(\mathcal{M})$, the fundamental group of $\mathcal{M}$. We have the hexagon $H_{1}$ whose sides $s_{i}$ are the arcs of $\gamma_{i}$ and these arcs are contained in the axes of the hyperbolic transformations $h_{i}(i=1, \ldots, 6)$. Their translation length in the positive direction along these axis is $2 \ell_{i}$ where $\ell_{i}$ denotes the length of $\gamma_{i}=\ell\left(\gamma_{i}\right)$. They are four of the parameters. The other parameters are the gluing angles. So

$$
\begin{aligned}
& c_{1}=\ell\left(\gamma_{1}\right), \quad c_{2}=\ell\left(\gamma_{2}\right), \quad c_{3}=\ell\left(\gamma_{3}\right), \quad c_{4}=\ell\left(\gamma_{4}\right), \\
& \sigma=\left|P-P_{2}\right|, \quad \tau=\operatorname{arctanh}\left(\cos \left(\theta_{2}\right)\right), \rho=\operatorname{arctanh}\left(\cos \left(\theta_{3}\right)\right) .
\end{aligned}
$$

But $c_{4}$ is determined by the others parameters, so with this parametrization, each point $t_{i}$ of the Teichmüller space $\mathcal{T}$ is $t_{i}=t_{i}\left(c_{1}, c_{2}, c_{3}, \sigma, \tau, \rho\right)$. This construction is dependent from de choice of the original geodesics $\gamma_{i}$; thus the dependence from the parameters $c_{i}=\ell\left(\gamma_{i}\right)$.

The sides are labeled $s_{1}, \ldots, s_{12}$ reading counterclockwise from zero.

Let $H^{2} / \Gamma$ our compact surface of genus $g=2$. The fundamental domain is a bounded fundamental polygon whose boundary $\partial F$ consists of the 12 geodesics segments $s_{1}, \ldots, s_{12}$.

Each side $s_{i}$ of $F$ is identified with $s_{j}$, by an element $g \in \Gamma$ and so each $g \in \Gamma$ produces a unique side $s$, namely, $s=\bar{F} \cap g(\bar{F})$. There is a bijection between the set of the sides of $F$ and the set of elements $g$ in $\Gamma$ for which $\bar{F} \cap g(\bar{F})$ is a side of $F$.

We construct a map from the set of the sides of $F$ onto itself, $g: s_{i} \rightarrow s_{j}$ where $s_{i}$ is identified with $s_{j}$. This is called a side-pairing of $F$. The side-pairing elements of $\Gamma$ generate $\Gamma$.

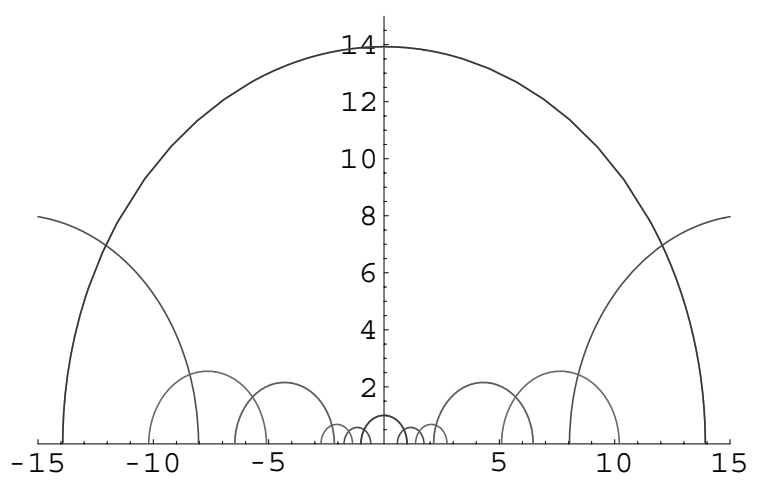

Figure 2. Fundamental polygon in the hyperbolic plane. 
In this construction, we choose the side rule for the pairing

$$
\begin{aligned}
& s_{1} \rightarrow s_{7}, \quad s_{2} \rightarrow s_{12}, \quad s_{3} \rightarrow s_{5}, \\
& s_{4} \rightarrow s_{10}, \quad s_{6} \rightarrow s_{8}, \quad s_{9} \rightarrow s_{11} .
\end{aligned}
$$

With this choice we explicitly calculate formulas for the side pairing transformations $g_{1}, \ldots, g_{6}$, $g_{7}=g_{1}^{-1}, \ldots, g_{12}=g_{6}^{-1}$. This means that

$$
\begin{aligned}
& s_{1}=g_{7}\left(s_{7}\right), \ldots, s_{11}=g_{12}\left(s_{9}\right) \\
& s_{7}=g_{1}\left(s_{1}\right), \ldots, s_{9}=g_{6}\left(s_{11}\right) .
\end{aligned}
$$

Thus, we obtain explicitly the generators $g_{i}=g_{i}\left(c_{1}, c_{2}, c_{3}, \sigma, \tau, \rho\right), i=1, \ldots, 12$. For easier computation we denote the Fenchel-Nielsen coordinates by $\ell_{1}=c_{1}, \ell_{2}=c_{2}, \ell_{3}=c_{3}, \ell_{4}=\sigma, \ell_{5}=\tau$ and $\ell_{6}=\rho$.

With the linear fractional transformations defined above it is possible to obtain the boundary map: $f_{\Gamma}: \partial F \rightarrow \partial F$, defined by piecewise linear fractional transformations in the partition $P=\left\{I_{i}=\right.$ $\left.\left[p_{i}, p_{i+1}\right), i=1, \ldots, 11\left[p_{12}, p_{1}\right)\right\}$, which is orbit equivalent to the action of the fundamental group $\Gamma$ on $\partial F$. The boundary map is represented by

$$
\begin{aligned}
& f_{\Gamma}: \bigcup_{i=1, \ldots, 12} I_{i} \rightarrow \bigcup_{i=1, \ldots, 12} I_{i} \\
& f_{\Gamma}(x)_{\mid I_{i}}=g_{i}(x), \quad i=1, \ldots, 12
\end{aligned}
$$

We are able to define a map that codifies the expansion of boundary points of $F$. And we determine the Markov matrix $A_{\Gamma}$ associated to $\Gamma$. Let be $A_{\Gamma}$ the matrix

$$
a_{i j}=\left\{\begin{array}{ll}
1 & \text { if } J_{j} \subset f_{\Gamma}\left(J_{i}\right) \\
0 & \text { otherwise }
\end{array} .\right.
$$

In [8], we have introduced a Markov partition for the Bowen-Series boundary map $f_{\Gamma}$ associated with the fundamental group $\Gamma$ and we defined the $24 \times 24$ Markov matrix $A_{\Gamma}$. The topological description is collected in this matrix.

It is known that there is a correspondence between the closed geodesics of the surface and the conjugacy classes of the group, so we can identify each closed geodesic. We obtained the length spectrum of the closed geodesics by computing

$$
\ell(g)=2 \operatorname{Arccosh}(\operatorname{tr}(g) / 2)
$$

As $A_{\Gamma}$ matrix codifies every information about the number of geodesics, we can introduce another matrix, also with $24 \times 24$ elements, that depends on the length spectrum of the geodesics, a weighted finite matrix $W$. The weights are defined by the geometrical invariants of the model. Let be $W$ the matrix:

$$
\begin{aligned}
w_{i j} & =\left(e^{-s \ell\left(g_{k}\right)} a_{i j}\right) \\
i, j & =1, \ldots, 24 \\
k & =1, \ldots, 12
\end{aligned}
$$


We have the framework to use the symbolic dynamics.

By this method, returning to our original question, we can study the variation of the first eigenvalue of the Laplacian with the Fenchel-Nielsen coordinates $\left(\ell_{1}, \ell_{2}\right.$, and $\left.\ell_{3}\right)$ that characterize the hyperbolic surface (see Figure 3).

We are able to introduce our main result about the conductance of the system. But first we need some definitions.

Definition 3. Let there be a geodesic chain $\gamma_{1}, \gamma_{2}, \gamma_{3}, \gamma_{4}$ where the four geodesics have equal length and the twist parameters are zero. We call regular domain of the genus $g=2$ the closed Riemann surface $\mathcal{M}$ with this Fenchel-Nielsen coordinates choice.

Definition 4. Let be $\beta\left(\ell_{0}\right)$ the conductance when it is considered the regular domain.

Theorem 5. Let $M$ be a closed Riemannian manifold, $\beta\left(\ell_{i}\right)$ the conductance on $M$ and $\ell_{i}$ the FenchelNielsen coordinate. Then, $\beta\left(\ell_{i}\right) \leq \beta\left(\ell_{0}\right)$.

So the regular case is when we have the maximum of the conductance. This result can be proved by symbolic dynamic methods and it is illustrated by Figures 4 and 5 .

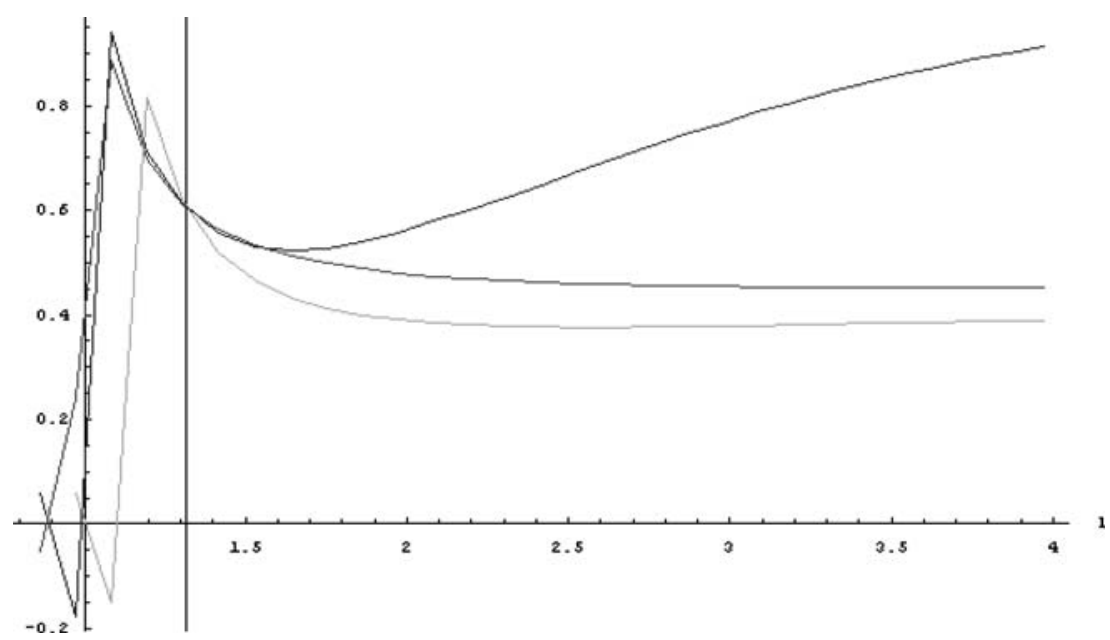

Figure 3. Variation of the first eigenvalue of the Laplacian with the Fenchel-Nielsen coordinates $\ell_{1}, \ell_{2}$, and $\ell_{3}$.

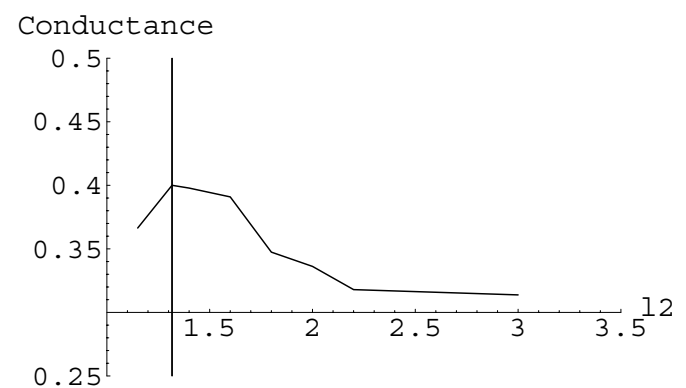

Figure 4. Variation of conductance with the Fenchel-Nielsen coordinate $\ell_{2}$. 


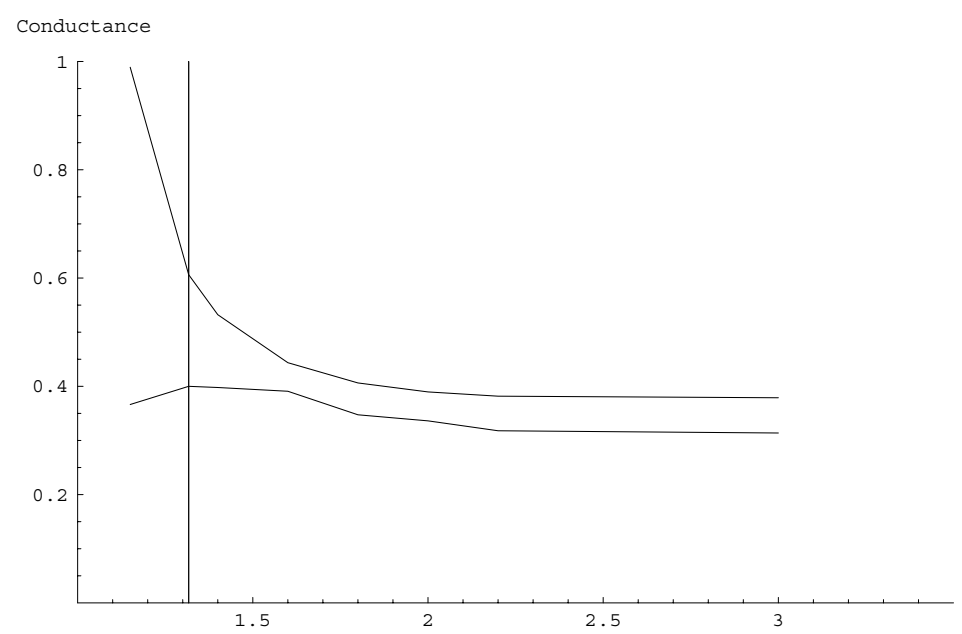

Figure 5. Variation of conductance and the first eigenvalue with the Fenchel-Nielsen coordinate $\ell_{2}$.

We compute the conductance and the first eigenvalue of the Laplacian for the different values of the Fenchel-Nielsen parameters and obtain the relationship between the smallest non-zero eigenvalue of the Laplacian and the conductance of the dynamical system.

\section{Acknowledgement}

The authors are (partially) supported by FCT (Portugal) through the program POCTI/FEDER.

\section{References}

1. Selberg, A., 'On the estimation of Fourier coefficients of modular forms', in Theory of Numbers, Proceedings of the Symposium on Pure Mathematics, Vol. 8, A. L. Whiteman (ed.), 1965, American Mathematical Society, Providence RI, 1965, pp. 1-15.

2. Grácio, C. and Sousa Ramos, J., 'Boundary maps and Fenchel-Nielsen coordinates', International Journal of Bifurcation and Chaos 13(7), 2003, 1949-1958.

3. Cheeger, J., 'A lower bound for the smallest eigenvalue of the Laplacian', in Problems in Analysis, Gunning (ed.), Princeton University Press, Princeton, 1970, pp. 195-199.

4. Buser, P., 'Cubic graphs and the first eigenvalue of a Riemann surface', Mathematische Zeitschrift 162, 1978, 87-99.

5. Buser, P., 'A note on the isoperimetric constant', Annales Scientifiques de l École Normale Superieure 15, 1982, $213-230$.

6. Brooks, R., 'Spectral geometry and the Cheeger constant', In Friedman, J. (ed.), in Expanding Graphs, Proceedings of the DIMACS Workshop, American Mathematical Society, 1993, pp. 5-19.

7. Fernandes, S. and Sousa Ramos, J., 'Spectral invariants of iterated maps of the interval', Grazer Mathematische Berichte 346, 2004, 113-122

8. Grácio, C. and Sousa Ramos, J., 'Symbolic dynamics and hyperbolic groups', Grazer Mathematische Berichte 339, 1999, 195-206. 